\title{
MoM Impedance Integrals in Conductive Media
}

\author{
Joris Peeters* ${ }^{* \dagger}$, Ignace Bogaert*, Kristof Cools ${ }^{* \ddagger}$ and Daniël de Zutter* \\ * Department of Information Technology \\ Ghent University, Ghent, Belgium \\ ${ }^{\dagger}$ Corresponding author \\ Email: joris.peeters@intec.ugent.be \\ ${ }_{\ddagger}^{\ddagger}$ Presenting author
}

\begin{abstract}
During the past decades, much research has been done towards the efficient calculation of impedance integrals in the Method of Moments. However, these results were almost always uniquely concerned with penetrable media. In this contribution, it will be shown how the integrals can be treated in highly conductive media as well. The rapid exponential decline of the Green's function, due to the losses, is the root of all additional complexities. The method as presented here takes care of these problems in a scalable way, i.e. the computation time becomes independent of the conductivity of the material. It is not meant as a replacement for techniques in penetrable media, due to some additional costs, but is - to our knowledge - the only approach that currently exists to efficiently handle conductive media. This paper presents the ideas and techniques in a fairly condensed manner. More information can be found in [1].
\end{abstract}

\section{INTRODUCTION}

The impedance integrals in the Method of Moments describe the interaction between basis and test functions by means of the Green's function $\frac{e^{-j k r}}{4 \pi r}$ or its gradient, with $k$ the wave number in the interaction domain and the wavelength given by $\lambda=\frac{2 \pi}{k}$. In penetrable media the losses are small or negligible and the numerator of the Green's function, $e^{-j k r}=\cos (k r)-j \sin (k r)$, is well-behaved. As such, most of the past and current research has focused on the singular $\frac{1}{r}$ behaviour and how to efficiently evaluate the impedance integrals when basis and test functions are close to each other (or even coinciding). The two most important techniques to deal with the singularity are Singularity Cancellation (SC) and Singularity Extraction (SE), which will be briefly revisited later. However, in highly conductive media, the wave number is approximately given by $k \approx \frac{i-j}{\delta}$, with the skin depth $\delta=\sqrt{\frac{2}{\omega \mu \sigma}}$ and $\sigma$ denoting the conductivity. Consequently, as the skin depth decreases, the previously well-behaved numerator now becomes wildly oscillatory and very strongly damped. This leads to new issues with regard to efficient quadrature schemes, that have not previously been treated in literature except for [2]. The novelty of our approach is scalability for both the inner (basis) and outer (test) integrals, making it of large practical use. The outline of this paper is as follows. Section II gives a brief overview of those integrals that need to be calculated and how the singular cases are dealt with in the case of penetrable media. Section III introduces our new approach and, finally, Section IV demonstrates how our approach could be useful by means of a numerical example.

\section{IMPEDANCE INTEGRALS IN MOM}

Assuming RWG expansion functions, the integrals that need to be calculated in order to fill the impedance matrix can be written as [1],

$$
\begin{gathered}
I_{t 1}=\int_{S_{i}} \boldsymbol{r} \cdot \int_{S_{j}} g(R) \boldsymbol{r}^{\prime} \mathrm{d} S^{\prime} \mathrm{d} S \\
I_{t 2}=\int_{S_{i}} \int_{S_{j}} g(R) \mathrm{d} S^{\prime} \mathrm{d} S \\
\boldsymbol{I}_{t 3}=\mathrm{PV} \int_{S_{i}} \int_{S_{j}} \nabla g(R) \times \boldsymbol{r}^{\prime} \mathrm{d} S^{\prime} \mathrm{d} S
\end{gathered}
$$

with others merely being variations in the presence or absence of $\boldsymbol{r}$ and $\boldsymbol{r}^{\prime}$ (with $R=\left|\boldsymbol{r}-\boldsymbol{r}^{\prime}\right|$ ). Here, $S_{i}$ and $S_{j}$ indicate the triangles for the observer (test) and source (basis) triangles. In penetrable media, these can easily be evaluated through, e.g., Gaussian quadrature when the supports are well-separated. When $S_{i}=S_{j}$ or they touch in a point or line, the integrands are singular. However, the integral can still be evaluated. The technique of Singularity Extraction [3],[4],[5],[6] subtracts the static singular part from the integral, until a regular integrand remains, although it is still discontinuous in its derivative. In order to obtain an integrand that is continuous in more derivatives, additional terms need to be extracted. These singular parts can be evaluated analytically. Alternatively, Singularity Cancellation [7],[8],[9], employs a coordinate transform through which the resulting Jacobian exactly cancels the singularity. Because it does not rely on analytical formulas, $\mathrm{SC}$ is more flexible, which could have advantages in the case of, e.g., higher order expansion functions or anisotropic media.

\section{Calculation in Conductive Media}

When trying to employ the typical techniques for penetrable media, i.e. SE and SC, but with Green's functions that result from conductive media, it appears that they both fail rather rapidly. The issues originate both in the inner and outer integrals. Due to the sharply peaked behaviour (exponentially damped) of the Green's function in conductive media, the inner integrand only contributes in a very small region that is closest to the observer point. Both SE and SC ignore this localised behaviour and fail to capture it accurately, often missing this contributing zone altogether. In addition to problems with the numerical quadratures, SE additionally suffers from severe cancellation issues between the analytical terms. In conclusion, 
SC generally leads to a vanishing result and SE to an exploding result for conductive media. The outer integral is less sensitive to error, although a certain effort is required to guarantee a scalable and accurate result. Without any specific techniques, relative accuracy would typically stall at $10^{-2}$ in the best case scenario but usually fail altogether. However, we will demonstrate how the outer integral as well can be controlled. In a first subsection, we will deal with the inner integral. The outer integral will be treated afterwards.

\section{A. Inner Integral}

Our explanation will be based on the integral

$$
I_{j}(\boldsymbol{r})=\int_{S_{j}} g\left(\left|\boldsymbol{r}-\boldsymbol{r}^{\prime}\right|\right) \mathrm{d} S^{\prime}
$$

although the techniques are equally applicable to similar integrals that feature powers of $\boldsymbol{r}^{\prime}$. The first step is the reduction of the integration domain to the region where the integrand is non-negligible. The numerator of the Green's function $g$ drops to a value $\epsilon_{c u t}$ after a distance given by,

$$
r_{c u t}=-\delta \ln \epsilon_{c u t}
$$

This distance will be used to define the new integration domain. As the conductivity grows, this domain will become smaller. That way the computational effort remains independent of $\sigma$ whilst still achieving the same accuracy. First of all, the integral is transformed to polar coordinates around a carefully chosen point $\boldsymbol{r}_{O}$, leading to the following form:

$$
I_{j}(\boldsymbol{r})=\int_{\phi_{1}}^{\phi_{2}} \mathrm{~d} \phi \int_{\rho_{1}(\phi)}^{\rho_{2}(\phi)} g\left(\left|\boldsymbol{r}-\left(\boldsymbol{r}_{0}+\rho \boldsymbol{u}_{\rho}\right)\right|\right) \rho \mathrm{d} \rho
$$

Note how this is essentially a Duffy transform from Singularity Cancellation. The projection point $\boldsymbol{r}_{O}$ is that point in the plane of $S_{j}$ that lies in the triangle (including the edges and corners) but is closest to the orthogonal projection of $\boldsymbol{r}$. Afterwards, the triangle $S_{j}$ is split such that $\boldsymbol{r}_{O}$ lies in a corner. This is demonstrated in Figure 1
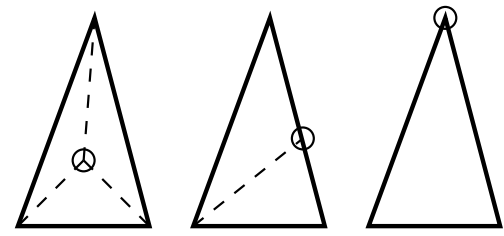

Fig. 1. The division into subtriangles for three different cases. The small circle indicates the location of $\boldsymbol{r}_{o}$ and is chosen as that point in the triangle that is closest to the orthogonal projection of the observation point into its plane.

Around the point $\boldsymbol{r}_{O}$, the integration domain can now be truncated in the radial direction, in such a way that the distance to the observation point is never greater than $r_{c u t}$. By limiting the radial integration in such a way, the spectral bandwidth of the remaining integrand is also limited, so the required number of quadrature points is controlled. In [1], both the Double Exponential [10], [11] and Gauss quadrature are discussed for
TABLE I

THE RELATIVE ERRORS AS A FUNCTION OF $k=q-i q$ FOR EVALUATION OF THE INNER INTEGRAL USING SINGULARITY EXTRACTION (WITH 15 TERMS), SINGULARITY CANCELLATION (WITH 17 QUADRATURE POINTS BOTH FOR THE RADIAL PART AND THE ANGULAR PART) AND OUR NOVEL APPROACH (USING THE SAME AMOUNT OF QUADRATURE POINTS AS SC AND $r_{c u t}$ FOR A TOLERANCE OF $10^{-2}$ ).

\begin{tabular}{|c|c|c|c|}
\hline$q$ & $\epsilon_{\text {rel,SE }}$ & $\epsilon_{\text {rel,SC }}$ & $\epsilon_{\text {rel, novel }}$ \\
\hline $10^{0}$ & $3 \cdot 10^{-7}$ & $2 \cdot 10^{-7}$ & $2 \cdot 10^{-7}$ \\
$10^{1}$ & $1 \cdot 10^{-6}$ & $3 \cdot 10^{-7}$ & $5 \cdot 10^{-4}$ \\
$10^{2}$ & $3 \cdot 10^{29}$ & $5 \cdot 10^{-5}$ & $1 \cdot 10^{-2}$ \\
$10^{3}$ & $4 \cdot 10^{69}$ & $8 \cdot 10^{-1}$ & $1 \cdot 10^{-2}$ \\
$10^{4}$ & $4 \cdot 10^{109}$ & $1 \cdot 10^{0}$ & $1 \cdot 10^{-2}$ \\
\hline
\end{tabular}

the integration, but the difference is small. The angular integral can be handled with Gauss quadrature rules, using a small amount of sampling points (provided the conductivity is indeed very large).

Table I shows the comparison between relative errors for the different methods. Clearly, our approach is the only one that maintains the desired accuracy as the conductivity increases.

\section{B. Outer Integral}

The outer integral is defined as

$$
I_{i j}=\int_{S_{i}} I_{j}(\boldsymbol{r}) \mathrm{d} S
$$

In order to demonstrate the potential issues, imagine two orthogonal triangles that have one edge in common (the so-called neighbour patch) and a skin depth that is much smaller than the characteristic size of these triangles. Clearly, the outer integrand will be zero almost everywhere on the triangle, except in a small region close to the shared egde. Another interesting case is that of the self patch (test and basis triangle are identical). It is easily seen that the outer integrand is constant (and different from zero) on almost the entire triangle except near the edges. From these two special cases it becomes clear that large variations in the integrand only occur near regions where the surfaces end, e.g. near edges. The characteristic size of these regions is typically equal to $r_{c u t}$, with reference to a certain chosen tolerance. By defining the regions of strong variation in the integrand, the quadrature points can be suitably focused and as such an accurate evaluation can be obtained with minimal effort. In order to do this, a number of cuts and projections is used, that eventually results in a break-up of the outer integration domain into a number of smaller domains, each covering a region where rapid variation might occur. For more information with regard to these projections, the reader is referred to [1], which also includes further numerical tests. These tests indicate that the accuracy of the outer integral can indeed be maintained in a scalable manner, i.e. independent of the conductivity. Combining the techniques for inner and outer integral, we have a method at our disposal that can handle the impedance integrals for highly conductive media in an efficient, accurate and controllable way. 


\section{NUMERICAL EXAMPLE}

More often than not, simulations that involve highly conductive materials can be done in an approximative way by assuming either a perfectly conducting material or by using a surface impedance. Naturally, these simulations do not require the previously described methods. However, there is a growing class of examples that could strongly benefit from an accurate modelling of the inside of the conductor. To mention just a few, one could study the presence of impurities inside the conductor or the interaction between both sides if it is very thin. Near corners a much finer geometrical meshing is required, which, to a certain extent, reduces the calculation of impedance integrals to the traditional methods of SE or SC. However, as the geometry becomes smoother, our technique allows the meshing to be considerably coarser (roughly $\frac{\lambda}{10}$ for the outside medium) and consequently save a huge amount of computational resources. The numerical example shown here features a very thin conductor shaped as a spherical shell, shown in Figure 2. The advantage of this particular geometry is that it allows comparison with an analytical solution for embedded spheres. The radius of the inner sphere is $1 \mathrm{~m}$ and

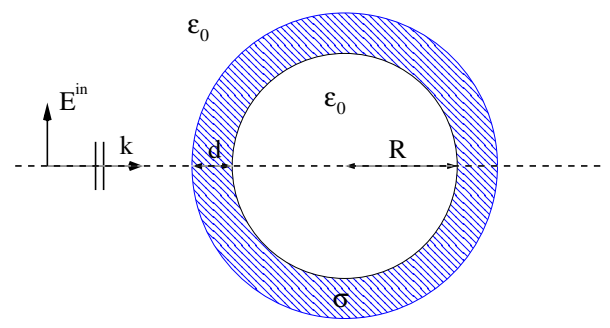

Fig. 2. The geometry for the numerical example.

the thickness of the material is $10 \mu \mathrm{m}$. The material is copper, such that $\sigma=59.6 \cdot 10^{6} \mathrm{~S} \cdot \mathrm{m}^{-1}$. An incoming plane wave with frequency $4.77 \cdot 10^{7} \mathrm{~Hz}$ impinges on this object. The skin depth of copper at this frequency is $\delta=9.46 \mu \mathrm{m}$ and the surface of each sphere is discretised in 584 triangles. This results in a linear system with 3504 degrees of freedom. The tolerance for the impedance integrals was set to $10^{-5}$. This particular example features a number of interesting cases, i.e. all the singular ones (self patch, neighbour patch and point patch), but also the common case of parallel (but non-touching) triangles. A comparison between the calculated total fields is shown in Figure 3, along a line through the center of the spheres and parallel to the direction of the plane wave.

Clearly excellent agreement is achieved, both inside and outside the shell. The only discrepancies occur very close to the interfaces and are due to geometrical error (the sphere is discretised with flat triangles). When using a perfectly electrically conducting shell or when using surface impedances, the field inside could not have been calculated. This demonstrates the applicability of our technique to tunnelling simulations.

\section{CONCLUSION}

This paper briefly explains the concepts used to calculate MoM impedance integrals in conductive media, without get-

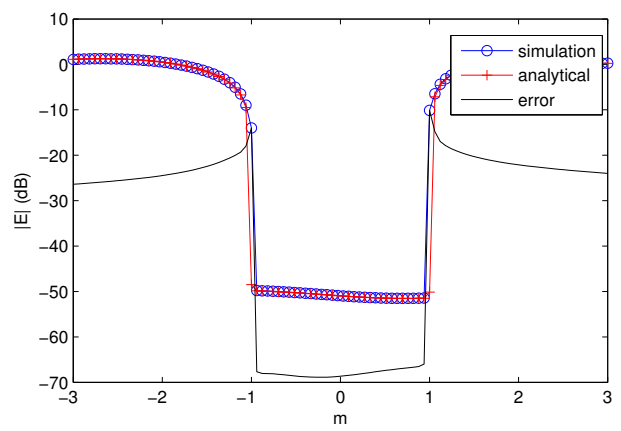

Fig. 3. A comparison (between simulation and analytical result) of the electric field after scattering at a very thin conductive shell. The difference (in $\mathrm{dB}$ ) between the error and solution graphs indicates the relative accuracy.

ting too deeply involved in details. The core of the method is the use of a truncation distance $r_{c u t}$, defined by the material parameters, frequency and desired tolerance. This allows suitable reductions of the integration domain, both for the inner and outer integral, allowing a scalable solution through the focusing of quadrature points. A number of suggestions for applications were given and one example, featuring tunnelling through a conductor, was shown to excellently agree with the analytical solution.

\section{REFERENCES}

[1] J. Peeters, I. Bogaert, and D. De Zutter, "Calculation of MoM Interaction Integrals in Highly Conductive Media," IEEE Trans. Antennas Propagation, 2010, under review.

[2] S. Chakraborty and V. Jandhyala, "Evaluation of Green's Function Integrals in Conducting media," IEEE Trans. Antennas Propagation, vol. 52, no. 12, pp. 3357-3363, 2004.

[3] D. Wilton, S. Rao, A. Glisson, D. Schaubert, O. Al-Bundak, and C. Butler, "Potential Integrals for Uniform and Linear Source Distributions on Polygonal and Polyhedral Domains," IEEE Trans. Antennas Propagation, vol. 32, no. 3, pp. 276-281, Mar. 1984.

[4] P. Ylä-Oijala and M. Taskinen, "Calculation of CFIE Impedance Matrix Elements with RWG and $n \times$ RWG Functions," IEEE Trans. Antennas Propagation, vol. 51, no. 8, pp. 1837-1846, Aug. 2003.

[5] R. Graglia, "On the Numerical Integration of the Linear Shape Functions Times the 3D-Green's Function or its Gradient on a Plane Triangle,' IEEE Trans. Antennas Propagation, vol. 41, no. 10, pp. 1448-1455, Oct. 1993.

[6] T. Eibert and V. Hansen, "On the Calculation of Potential Integrals for Linear Source Distributions on Triangular Domains," IEEE Trans. Antennas Propagation, vol. 43, no. 12, pp. 1499-1502, 1993.

[7] M. Khayat and D. Wilton, "Numerical Evaluation of Singular and Near-Singular Potential Integrals," IEEE Trans. Antennas Propagation, vol. 53, no. 10 , pp. 3180-3190, Oct. 2005.

[8] L. Rossi and P. Cullen, "On the Fully Numerical Evaluation of the Linear-Shape Function Times the 3D Green's Function on a Plane Triangle," IEEE Trans. Microwave Theory Tech., vol. 47, no. 4, pp. 398-402, Apr. 1999.

[9] R. Graglia and G. Lombardi, "Machine Precision Evaluation of Singular and Near Singular Integrals by Use of Gauss Quadrature Formulas for Rational Functiuns," IEEE Trans. Antennas Propagation, vol. 56, no. 4, pp. 981-998, Apr. 2008.

[10] A. Polimeridis and J. Mosig, "Evaluation of Weakly Singular Integrals Via Generalized Cartesian Product Rules Based on the Double Exponential Formula," IEEE Trans. Antennas Propagation, vol. 58, no. 6, pp. 1980-1988, Jun. 2010.

[11] M. Mori, "Discovery of the Double Exponential Transformation and Its Developments," Publ. RIMS, Kyoto Univ., vol. 41, pp. 897-935, 2005. 